\title{
On Local Generalized Ulam-Hyers Stability for Nonlinear Fractional Functional Differential Equation
}

\author{
Dongming Nie, ${ }^{1}$ Azmat Ullah Khan Niazi $\mathbb{D D}^{2,3}$ and Bilal Ahmed ${ }^{3}$ \\ ${ }^{1}$ Department of Common Courses, Anhui Xinhua University, Hefei 230088, China \\ ${ }^{2}$ Department of Mathematics, Xiangtan University, Xiangtan, Hunan 411105, China \\ ${ }^{3}$ Department of Mathematics and Statistics, University of Lahore, Sargodha, Lahore, Pakistan \\ Correspondence should be addressed to Azmat Ullah Khan Niazi; azmatniazi35@gmail.com
}

Received 27 April 2020; Accepted 27 May 2020; Published 18 June 2020

Guest Editor: Shaohui Wang

Copyright (c) 2020 Dongming Nie et al. This is an open access article distributed under the Creative Commons Attribution License, which permits unrestricted use, distribution, and reproduction in any medium, provided the original work is properly cited.

We discuss the existence of positive solution for a class of nonlinear fractional differential equations with delay involving Caputo derivative. Well-known Leray-Schauder theorem, Arzela-Ascoli theorem, and Banach contraction principle are used for the fixed point property and existence of a solution. We establish local generalized Ulam-Hyers stability and local generalized Ulam-Hyers-Rassias stability for the same class of nonlinear fractional neutral differential equations. The simulation of an example is also given to show the applicability of our results.

\section{Introduction}

Fractional differential equations (FDEs) have boosted considerably due to their application in various fields of sciences, such as engineering, chemistry, mechanics, and physics. Recently, fractional ordinary differential equations (ODEs) and partial differential equations (PDEs) have been developed significantly. Indeed, we can also find applications in control, electromagnetism, and electrochemistry (see $[1,2])$. For more details in this area, one can also see the monographs of Kilbas et al. [3], Li et al. [4, 5], Liu et al. [6-9], Miller and Ross [10], Podulbny [11], Rehman et al. [12], and the references therein.

Positive solution of fractional ODEs have already discussed in [13-15]. However, until now research on existence of positive solution for fractional functional differential equations (FFDEs) is rare. Research in different fields including engineering, physics, and biosciences have proved that numerous system structures explain more exactly with the help of FDEs [16-21] and similarly FDEs with delay [22-27] are more accurate to illustrate the real world problems compared to FDEs without delay. So, in [28], Miller and Ross mentioned that this field has been touched by almost all fields of engineering and science.
Idea of Ulam stability started in 1940, when during the talk at Wisconsin University Ulam posed a problem that "When can we assert that an approximate solution of a functional equation can be approximated by a solution of the corresponding equation?" (for more details, see [29]). After one year, Hyers first gave the answer to the Ulam's question [30] in case of Banach spaces. After that, stability of this type is called the Ulam-Hyers stability. Rassias [31] in 1978 provided an outstanding generalization of the Ulam-Hyers stability of mappings by considering variables.

Usually, the discussion of the existence and uniqueness of a solution for nonlinear FDEs normally fixed point theory has been used [16, 32, 33]. Motivated by these and [34-36], in this work, we have discussed existence and uniqueness of solution also after applying some sufficient conditions, obtained positive solution, and at the end established local generalized Ulam-Hyers stability and local generalized Ulam-Hyers-Rassias stability and presented stability results graphically for the class of nonlinear FDEs with delay given by

$$
\begin{cases}{ }^{c} D_{0}^{\gamma}\left[z(t)-g\left(t, z_{t}\right)\right]=z(t) f\left(t, z_{t}\right), & t \in I, \\ z(t)=\psi(t) \geq 0, & t \in[-\tau, 0],\end{cases}
$$


where ${ }^{c} D_{0}^{\gamma}$ is the Caputo derivative and $0<\gamma<1$, while $\psi \in C$ and $I=[0, T]$ where $C=C\left([-\tau, 0] ; \mathbb{R}^{+}\right)$is space of continuous functions, $\tau$ is a nonnegative real number also $f, g \in\left(I \times C, \mathbb{R}^{+}\right)$are continuous functions, and $z_{t} \in C$ is defined as $z_{t}(\theta)=z(t+\theta),-\tau \leq \theta \leq 0$.

Remaining paper is arranged as follows. Section 2 includes some basic definitions and lemmas used throughout this paper. In Section 3, we have the main results. In Section 4, we establish local generalized Ulam-Hyers stability and local generalized Ulam-Hyers-Rassias stability for problem (1), and in Section 5, simulation of an example is given to show the applicability of our results.

\section{Preliminaries}

Let $Q$ be a cone in a real Banach space $X$ and partial order $\leq$ introduced by $Q$ in $X$ is as

$$
\text { if } v-u \in Q \text { then } u \leq v \text {. }
$$

Definition 1. Let $u, v \in X$ be the order interval $\langle u, v\rangle$ defined as

$$
\langle u, v\rangle=\{w \in X: u \leq w \leq v\} .
$$

Definition 2. The functional $h\left(t, z_{t}\right)$ is said to be nondecreasing w.r.t. $\phi$ on $I \times C$ such that

$$
x(\phi) \leq y(\phi), \quad \phi \in[-\tau, 0],
$$

implies that

$$
h(t, x) \leq h(t, y)
$$

for any $(t, x)$ and $(t, y) \in I \times C$.

Definition 3 (see [11]). The fractional integral of order $\gamma$ for a function $g$ with lower limit 0 can be defined as

$$
I_{0}^{\gamma} g(t)=\frac{1}{\Gamma(\gamma)} \int_{0}^{t} \frac{g(s)}{(t-s)^{1-\gamma}} \mathrm{d} s, \quad \gamma>0, t>0,
$$

where $\Gamma$ is the gamma function and right-hand side of upper equality is defined pointwise on $\mathbb{R}^{+}$.

Definition 4 (see [11]). The left Caputo fractional derivative of order $\gamma$ is given by

$$
{ }^{c} D_{t}^{\gamma} f(t)=\frac{1}{\Gamma(n-\gamma)} \int_{a}^{t} \frac{f^{(n)}(s)}{(t-s)^{\gamma+1-n}} \mathrm{~d} s,
$$

where $n=[\gamma]+1([\gamma]$ stands for the bracket function of $\gamma)$.

Lemma 1 (Leray Schauder fixed point theorem, see [11]). Let $S_{r}$ be a nonempty, closed, bounded, and convex subset of Banach space $X$ and $Q: S_{r} \longrightarrow S_{r}$ is a compact and continuous map; then, $Q$ has a fixed point in $S_{r}$.

Theorem 1. If $U \subset Q$, where $Q$ is a cone of space of partial order $X, F: U \longrightarrow X$ be nondecreasing. If there exists $u_{0}, v_{0} \in U$ such that $v_{0} \geq u_{0},\left\langle u_{0}, v_{0}\right\rangle \subset U$, and $u_{0}$ is lower solution and $v_{0}$ is an upper solution of $y-F(y)=0$, then
$y-F(y)=0$ has minimum solution $u^{*}$ and maximum solution $v^{*}$ in $\left\langle u_{0}, v_{0}\right\rangle$ such that $v^{*} \geq u^{*}$, and if anyone of these conditions holds

(i) $X$ is reflexive, $F$ is weak continuous or continuous, and $Q$ is normal

(ii) $F$ is completely continuous and $Q$ is normal

(iii) $F$ is continuous and $Q$ is regular

\section{Main Results}

In this section, we have discussed existence and uniqueness of solution and some conditions for positive solution of equation (1). Consider $X=C[-\tau, T]$ is a Banach space endowed with the supremum norm and cone $Q$ is defined as $Q=\{z \in Q ; z(t) \geq 0,-\tau \leq t \leq T\}$. From equation (1), we have

$$
I_{0}^{\gamma}\left({ }^{c} D_{0}^{\gamma}\left[z(t)-g\left(t, z_{t}\right)\right]\right)=I_{0}^{\gamma}\left[z(t) f\left(t, z_{t}\right)\right],
$$

and we can easily get that

$$
\begin{aligned}
z(t)= & \psi(0)-g(0, \psi)+g\left(t, z_{t}\right) \\
& +\frac{1}{\Gamma(\gamma)} \int_{0}^{t}(t-\tau)^{\gamma-1} z(\tau) f\left(\tau, z_{\tau}\right) \mathrm{d} \tau, \quad t \in[0, T]
\end{aligned}
$$

Let $x(\cdot):[-\tau, T] \longrightarrow \mathbb{R}^{+}$be the function defined by

$$
x(t)= \begin{cases}\psi(t), & t \in[-\tau, 0], \\ 0, & t \in I .\end{cases}
$$

So, $x_{0}=\psi$ and for every $w \in C\left(I, \mathbb{R}^{+}\right)$with $w(0)=0$, and we defined $\bar{w}$ as

$$
\bar{w}(t)= \begin{cases}0, & t \in[-\tau, 0] \\ w(t), & t \in I\end{cases}
$$

Let $x(\cdot)$ satisfy equation (9), and we can decompose $z$ (.) as $z(t)=\bar{w}(t)+x(t)$, for $0 \leq t \leq T$ so that $z_{t}=\bar{w}_{t}+x_{t}$, for every $0 \leq t \leq T$, where $\bar{w}(t)$ is such that $|\bar{w}(t)|<1, \forall t \in I$ and the function $w(\cdot)$ satisfies

$$
\begin{aligned}
\bar{w}(t)= & \psi(0)-g(0, \psi)+g\left(t, \bar{w}_{t}+x_{t}\right) \\
& +\frac{1}{\Gamma(\gamma)} \int_{0}^{t}(t-s)^{\gamma-1}(\bar{w}(s)+x(s)) f\left(s, \bar{w}_{s}+x_{s}\right) \mathrm{d} s,
\end{aligned}
$$$$
t \in I \text {. }
$$

Set $H=\{z \in Q ; z=\psi(t), t \in[-\tau, 0]\} \subset Q$, where $Q$ is a cone and $Q \subset X$ and $X$ is a Banach space with Sup norm $\|\bar{w}(t)\| \leq \sup \{|w(t)| ;-\tau \leq t \leq T\}$. Let the operator $P: H \longrightarrow H$ be defined by

$$
\begin{aligned}
(P \bar{w})(t)= & \psi(0)-g(0, \psi)+g\left(t, \bar{w}_{t}+x_{t}\right) \\
& +\frac{1}{\Gamma(\gamma)} \int_{0}^{t}(t-s)^{\gamma-1} \bar{w}(s) f\left(s, \bar{w}_{s}+x_{s}\right) \mathrm{d} s .
\end{aligned}
$$


Before proving main results, we introduce the following conditions:

(C1) Let us take $f, g: I \times C \longrightarrow R^{+}$with $g(0, \psi)=0$, where function $g$ is nondecreasing and $\left|g\left(t, z_{t}\right)\right| \leq L_{1}$ and $f$ and $g$ satisfy Lipchitz condition that is, and there exists nonnegative constants $c_{1}$ and $c_{2}$ such that

$$
\begin{aligned}
& |f(t, u)-f(t, v)| \leq c_{1}\|u-v\|, \quad \forall t \in I, \text { and } u, v \in C, \\
& |g(t, u)-g(t, v)| \leq c_{2}\|u-v\|, \quad \forall t \in I, \text { and } u, v \in C .
\end{aligned}
$$

(C2) If $G \subset H$ be bounded, $f$ is nondecreasing and

$$
\max _{t \in I}\left\{f\left(t, z_{t}\right), z \in G\right\}=L-1,
$$

where $L>0$. Let $\{H=\{z \in Q ; z=\psi(t), t \in[-\tau, 0]\} \subset Q\}$. Define the operator $P$ by

$$
P \bar{w}(t)= \begin{cases}\psi(0)+g\left(t, \bar{w}_{t}+x_{t}\right)+\frac{1}{\Gamma(\gamma)} \int_{0}^{t}(t-s)^{\gamma-1} \bar{w}(s) f\left(s, \bar{w}_{s}+x_{s}\right) \mathrm{d} s, & t \in I, \\ 0, & t \in[-\tau, 0] .\end{cases}
$$

Theorem 2. Assume that $f$ and $g$ are nonnegative and continuous; then, operator $\mathrm{P}: H \longrightarrow H$ is completely continuous.

Proof. As we know that $f$ and $g$ are nonnegative and continuous, so operator $P: H \longrightarrow H$ is continuous. Let
$G \subset H$ is bounded, i.e., there exists a constant $l>0$ such that $\|z\| \leq l, \forall z \in G$. Here, we have to keep in mind the condition that $x(t)=0$ when $t \in I$. If $\max \left\{f\left(t, z_{t}\right) \mid 0 \leq t \leq T\right.$, $z \in G\}=L-1$, and for all $z \in G$, we get that

$$
\begin{aligned}
|(P \bar{w})(t)| & \leq\left|\psi+g\left(t, \overline{w_{t}}+x_{t}\right)+\frac{1}{\Gamma(\gamma)} \int_{0}^{t}(t-s)^{\gamma-1} \bar{w}(s) f\left(s, \bar{w}_{s}+x_{s}\right)\right| \mathrm{d} s \\
& \leq l+L_{1}+\frac{L}{\Gamma(\gamma)} \int_{0}^{t}(t-s)^{\gamma-1} \mathrm{~d} s \\
& \leq l+L_{1}+\frac{L T^{\gamma}}{\Gamma(\gamma+1)}
\end{aligned}
$$

Hence, $P G$ is bounded. Now, we show that $P G$ is equicontinuous, and the proof is divided into three possible steps.
Step 1: for every $\bar{w} \in G$ and $t_{1}, t_{2} \in I$ such that $t_{2}>t_{1}$, then

$$
\begin{aligned}
&\left|P \bar{w}\left(t_{1}\right)-P \bar{w}\left(t_{2}\right)\right| \leq\left|g\left(t_{1}, \bar{w}_{t_{1}}+x_{t_{1}}\right)-g\left(t_{2}, \bar{w}_{t_{2}}+x_{t_{2}}\right)\right|+\frac{1}{\Gamma(\gamma)} \int_{0}^{t_{1}}\left(t_{1}-s\right)^{\gamma-1}\left|\bar{w}(s) f\left(s, \bar{w}_{s}+x_{s}\right)\right| \mathrm{d} s \\
& \quad-\frac{1}{\Gamma(\gamma)} \int_{0}^{t_{2}}\left(t_{2}-s\right)^{\gamma-1}\left|\bar{w}(s) f\left(s, \bar{w}_{s}+x_{s}\right)\right| \mathrm{d} s \\
& \leq c_{2}\left\|\bar{w}_{t_{1}}-\bar{w}_{t_{2}}\right\|+\frac{1}{\Gamma(\gamma)}\left[\int_{0}^{t_{1}}\left(t_{1}-s\right)^{\gamma-1}-\int_{0}^{t_{2}}\left(t_{2}-s\right)^{\gamma-1}\right]\left|\bar{w}(s) f\left(s, \bar{w}_{s}+x_{s}\right)\right| \mathrm{d} s \\
& \leq c_{2}\left\|\bar{w}_{t_{1}}-\bar{w}_{t_{2}}\right\|+\frac{1}{\Gamma(\gamma)}\left|\bar{w}(t) \| f\left(t, \bar{w}_{t}+x_{t}\right)\right|\left[\int_{0}^{t_{1}}\left|\left(t_{1}-s\right)^{\gamma-1}-\left(t_{2}-s\right)^{\gamma-1}\right| \mathrm{d} s-\int_{t_{1}}^{t_{2}}\left(t_{2}-s\right)^{\gamma-1} \mathrm{~d} s\right] \\
& \leq c_{2}\left\|\bar{w}_{t_{1}}-\bar{w}_{t_{2}}\right\|+\frac{L}{\Gamma(\gamma+1)}\left(t_{1}^{\gamma}+\left(t_{2}-t_{1}\right)^{\gamma}-t_{2}^{\gamma}+\left(t_{2}-t_{1}\right)^{\gamma}\right) \\
&< c_{2} \sup \left\{\left|w\left(t_{1}\right)-w\left(t_{2}\right)\right|\right\}+\frac{L}{\Gamma(\gamma+1)}\left(t_{2}-t_{1}\right)^{\gamma} .
\end{aligned}
$$


As $t_{1} \longrightarrow t_{2}$, the right-hand side of above inequality tends to zero.

Step 2: for every $\bar{w} \in G$, as $\psi \in C[-\tau, 0]$ and $t_{1} \in[-\tau, 0)$ while $0 \leq t_{2} \leq T$, there exists $\delta>0$ such that when $\delta_{1}>t_{1},\left|\psi\left(t_{1}\right)-\psi(0)\right|<\epsilon / 2$.
If $\delta=\min \left\{\delta_{1},\left(\Gamma(\gamma+1)\left(\epsilon-2 L_{1} / 2 L\right)\right)^{1 / \gamma}\right\}$, then for $t_{2}-t_{1}<\delta$, we get that

$$
\begin{aligned}
\left|P \bar{w}\left(t_{1}\right)-P \bar{w}\left(t_{2}\right)\right| & \leq|\psi(t)-\psi(0)|+\left|g\left(t_{2}, \bar{w}_{t_{2}}+x_{t_{2}}\right)\right|+\frac{1}{\Gamma(\gamma)} \int_{0}^{t_{2}}\left(t_{2}-s\right)^{\gamma-1}\left|\bar{w}(s) f\left(s, \bar{w}_{s}+x_{s}\right)\right| \mathrm{d} s \\
& \leq \frac{\epsilon}{2}+L_{1}+\frac{L}{\Gamma(\gamma+1)} t_{2}^{\gamma}=\frac{\epsilon}{2}+L_{1}+\frac{L}{\Gamma(\gamma+1)} \delta^{\gamma} \leq \frac{\epsilon}{2}+\frac{\epsilon}{2}=\epsilon .
\end{aligned}
$$

Step 3: for every $\bar{w} \in G, \forall \epsilon>0$, and $t_{1}, t_{2} \in[-\tau, 0]$ where $t_{2}>t_{1}$. As $\psi$ is continuous, we have

$$
\left|P \bar{w}\left(t_{1}\right)-P \bar{w}\left(t_{2}\right)\right|=\left|\psi\left(t_{1}\right)-\psi\left(t_{2}\right)\right|<\epsilon \text {, when } \delta_{1}>t_{2}-t_{1} .
$$

Hence, $P G$ is equicontinuous. So, $\overline{P G}$ is compact by Arzela-Ascoli Theorem.

Theorem 3. Suppose that $C 1$ and $C 2$ hold. Let $f, g: I \times$ $C \longrightarrow \mathbb{R}^{+}$and $\left[c_{2}+\left(T^{\gamma} / \Gamma(\gamma+1)\right)\left(c_{1}+L\right)\right]<1$. Then, there exists a unique solution for IVP (1) on the interval $[-\tau, T]$.
Proof. Consider the operator $P: H \longrightarrow H$ defined as

$$
\begin{aligned}
(P \bar{w})(t)= & \psi(0)+g\left(t, \bar{w}_{t}+x_{t}\right) \\
& +\frac{1}{\Gamma(\gamma)} \int_{0}^{t}(t-s)^{\gamma-1} \bar{w}(s) f\left(s, \bar{w}_{s}+x_{s}\right) \mathrm{d} s .
\end{aligned}
$$

We will show that $P$ is a contraction. Let $\bar{w}_{1}, \bar{w}_{2} \in C\left(I, \mathbb{R}^{+}\right)$, then we obtain the following sequence of inequalities:

$$
\begin{aligned}
&\left|P \bar{w}_{1}(t)-P \bar{w}_{2}(t)\right| \leq\left|g\left(\begin{array}{c}
t, \bar{w}_{1 t} \\
+x_{t}
\end{array}\right)-g\left(t, \bar{w}_{2 t}+x_{t}\right)\right| \\
&+\left|\frac{1}{\Gamma(\gamma)} \int_{0}^{t}(t-s)^{\gamma-1} \bar{w}_{1}(s) f\left(s, \bar{w}_{1 s}+x_{s}\right) \mathrm{d} s-\frac{1}{\Gamma(\gamma)} \int_{0}^{t}(t-s)^{\gamma-1} \bar{w}_{2}(s) f\left(s, \bar{w}_{2 s}+x_{s}\right) \mathrm{d} s\right| \\
& \leq c_{2}\left\|\bar{w}_{1 t}-\bar{w}_{2 t}\right\|+\frac{1}{\Gamma(\gamma)} \int_{0}^{t}(t-s)^{\gamma-1}\left|\bar{w}_{1}(s) f\left(s, \bar{w}_{1 s}+x_{s}\right)-\bar{w}_{2}(s) f\left(s, \bar{w}_{2 s}+x_{s}\right)\right| \mathrm{d} s \\
& \leq c_{2}\left\|\bar{w}_{1 t}-\bar{w}_{2 t}\right\|+\frac{1}{\Gamma(\gamma)}\left[\int_{0}^{t}(t-s)^{\gamma-1}\left|\bar{w}_{1}(s) f\left(s, \bar{w}_{1 s}+x_{s}\right)-\bar{w}_{2}(s) f\left(s, \bar{w}_{2 s}+x_{s}\right)\right|\right. \\
& \leq c_{2}\left\|\bar{w}_{1 t}-\bar{w}_{2 t}\right\|+\frac{1}{\Gamma(\gamma)} \int_{0}^{t}(t-s)^{\gamma-1} \mid \bar{w}_{1}(s) f\left(s, \bar{w}_{1 s}+x_{s}\right)-\bar{w}_{1}(s) f\left(s, \bar{w}_{2 s}+x_{s}\right) \\
&+\bar{w}_{1}(s) f\left(s, \bar{w}_{2 s}+x_{s}\right)-\bar{w}_{2}(s) f\left(s, \bar{w}_{2 s}+x_{s}\right) \mid \mathrm{d} s \\
& \leq c_{2}\left\|\bar{w}_{1 t}-\bar{w}_{2 t}\right\|+\frac{1}{\Gamma(\gamma)}\left[\int_{0}^{t}(t-s)^{\gamma-1}\left|\bar{w}_{1 s}\right| c_{1}\left\|\bar{w}_{1 s}-\bar{w}_{2 s}\right\| \mathrm{d} s+\int_{0}^{t}(t-s)^{\gamma-1} L s u p\left|\left\{w_{1}(t)-w_{2}(t)\right\}\right| \mathrm{d} s\right] \\
& \leq c_{2} \sup \left\{\left|w_{1}(t)-w_{2}(t)\right|\right\}+\frac{c_{1} T^{\gamma}}{\Gamma(\gamma+1)} \sup \left\{\left|w_{1}(t)-w_{2}(t)\right|\right\}+\frac{L T^{\gamma}}{\Gamma(\gamma+1)} \sup \left\{\left|w_{1}(t)-w_{2}(t)\right|\right\} \\
& \leq {\left[c_{2}+\frac{T^{\gamma}}{\Gamma(\gamma+1)}\left(c_{1}+L\right)\right]\left\|\bar{w}_{1}-\bar{w}_{2}\right\| . }
\end{aligned}
$$


Therefore,

$$
\left\|P \bar{w}_{1}(t)-P \bar{w}_{2}(t)\right\| \leq\left[c_{2}+\frac{T^{\gamma}}{\Gamma(\gamma+1)}\left(c_{1}+L\right)\right]\left\|\bar{w}_{1}(t)-\bar{w}_{2}(t)\right\| .
$$

So, $P$ is a contraction, and hence by Banach's contraction principle $P$ has a unique fixed point.

Definition 5. The function $m \in X$ is said to be a lower solution of equation (1) (operator $P$ ) if

$$
\begin{cases}{ }^{c} D_{0}^{\gamma}\left[m(t)-g\left(t, m_{t}\right)\right] \leq m(t) f\left(t, m_{t}\right), & t \in I, \\ m(t) \leq \psi(t), & t \in[-\tau, 0],\end{cases}
$$

and the function $n \in X$ is said to be an upper solution of equation (1) if

$$
\begin{cases}{ }^{c} D_{0}^{\gamma}\left[n(t)-g\left(t, n_{t}\right)\right] \geq n(t) f\left(t, n_{t}\right), & t \in I, \\ n(t) \geq \psi(t), & t \in[-\tau, 0] .\end{cases}
$$

If inequalities are strict, then $m(t)$ and $n(t)$ are strict lower and upper solutions.

\section{Theorem 4. Let}

(H1) $f$ and $g$ from $I \times C$ to $\mathbb{R}^{+}$are continuous, and $f(t, \psi)$ and $g(t, \psi)$ are nondecreasing in $\psi$ for each $t \in I$

(H2) $m_{0}$ is lower and $n_{0}$ is an upper solution of equation (1), satisfying condition $m_{0}(t) \leq n_{0}(t), t \in I$ and $m_{0}, n_{0} \in H$

Then, equation (1) has at least a positive solution.

Proof. We only take fixed point operator $P$. By Theorem 2, $P$ is completely continuous and obviously by equation (16), $u_{0}$ and $v_{0}$ are lower and upper solutions of $P$, respectively. By (H1), $\bar{w}_{1}, \bar{w}_{2} \in H, \bar{w}_{1} \leq \bar{w}_{2}$, we have

$$
\begin{aligned}
P \bar{w}_{1}(t)= & \psi(0)+g\left(t, \bar{w}_{1 t}+x_{t}\right) \\
& +\frac{1}{\Gamma(\gamma)} \int_{0}^{t}(t-s)^{\gamma-1} \bar{w}_{1}(s) f\left(s, \bar{w}_{1 s}+x_{s}\right) \mathrm{d} s \\
\leq & \psi(0)+g\left(t, \bar{w}_{2 t}+x_{t}\right) \\
& +\frac{1}{\Gamma(\gamma)} \int_{0}^{t}(t-s)^{\gamma-1} \bar{w}_{2}(s) f\left(s, \bar{w}_{2 s}+x_{s}\right) \mathrm{d} s \\
= & P \bar{w}_{2}(t), \quad t \in I .
\end{aligned}
$$

So, the operator $P$ is nondecreasing. Also, we have

$$
\begin{aligned}
\operatorname{Pm}(t)= & \psi(0)+g\left(t, m_{t}+x_{t}\right) \\
& +\frac{1}{\Gamma(\gamma)} \int_{0}^{t}(t-s)^{\gamma-1} m(s) f\left(s, m_{s}\right) \mathrm{d} s,
\end{aligned}
$$

$P m(0)=\psi(0)+g\left(0, m_{0}+x_{0}\right) \geq m(0)$.

As $m \in X$ is a lower solution, therefore we can say that $P m(0) \geq m(0)$, similarly $P n(0) \leq n(0)$ from the definition of upper solution of $P$.

Hence, $P:\left\langle m_{0}, n_{0}\right\rangle \longrightarrow\left\langle m_{0}, n_{0}\right\rangle$ is a completely continuous operator. Since $Q$ is a normal cone, Theorem 1 implies that $P$ has a fixed point $\bar{w} \in\left\langle m_{0}, n_{0}\right\rangle$.

(H3) Here, we suppose that there exists $M>0$ such that $0<z f\left(t, z_{t}\right) \leq M, t \in I$.

Theorem 5. Assume conditions (H1) and (H3) holds; then, equation (1) has a positive solution.

Proof. Consider the equation

$$
\begin{cases}{ }^{c} D_{0}^{\gamma}\left[m(t)-g\left(t, m_{t}\right)\right]=0, & t \in I, \\ m(t)=\psi(t), & t \in[-\tau, 0] .\end{cases}
$$

Obviously, equation (28) has a solution $m(t)=m_{0}-g\left(t_{0}, \psi\right)+g\left(t, m_{t}\right)$, which is a lower solution of equation (1). Similarly, consider the equation

$$
\begin{cases}{ }^{c} D_{0}^{\gamma}\left[n(t)-g\left(t, n_{t}\right)\right]=M, & t \in I, \\ n(t)=\psi(t), & t \in[-\tau, 0] .\end{cases}
$$

We know that $n(t)=n_{0}-g\left(t_{0}, \psi\right)+g\left(t, n_{t}\right)+$ $\left(M t^{\gamma} / \Gamma(\gamma+1)\right), t \in I$ is an upper solution of equation (1) and $n(t) \geq m(t)$, so the above definition proves that equation (1) has minimum one positive solution.

For getting another result for a positive solution of equation (1), now we take the more general case of (1) and find the existence of a positive solution for the equations:

$$
\begin{cases}{ }^{c} D_{0}^{\gamma}\left[\nu(t)-g\left(t, v_{t}\right)\right]=N \nu(t), & t \in I . \\ \nu(t)=\psi(t), & t \in[-\tau, 0] .\end{cases}
$$

Theorem 6. Equation (30) has a least positive solution.

Proof. Equation (30) is equivalent to

$$
v(t)= \begin{cases}\nu(0)-g(0, \psi)+g(t, v(t))+\frac{1}{\Gamma(\gamma)} \int_{0}^{t}(t-s)^{\gamma-1} N \nu(s) \mathrm{d} s, & t \in I, \\ \psi(t), & t \in[-\tau, 0] .\end{cases}
$$


Let $P: H \longrightarrow H$ be an operator defined as

$$
P \nu(t)= \begin{cases}\nu(0)+g(t, v(t))+\frac{1}{\Gamma(\gamma)} \int_{0}^{t}(t-s)^{\gamma-1} N \nu(s) \mathrm{d} s, & t \in I, \\ \psi(t), & t \in[-\tau, 0] .\end{cases}
$$

$P$ is completely continuous by Theorem 2 .

Let us take $B_{R}=\left\{v(t) \in C\left([-\tau, T], \mathbb{R}^{+}\right) \mid\right.$ $\|\nu(t)-\nu(0)\| \leq R\}$ a bounded, closed, and convex subset of the Banach space $C[-\tau, \delta]$, where $\delta \in I$ and $R>0$. The possible two cases are

Case 1: for all $\nu \in B_{R}, t \in[I]$, we obtain

$$
\begin{aligned}
|P \nu(t)-v(0)| & \leq\left|g\left(t, v_{t}\right)\right|+\frac{1}{\Gamma(\gamma)} \int_{0}^{t}(t-s)^{\gamma-1}|N \nu(s)| \mathrm{d} s \\
& \leq L_{1}+\frac{N}{\Gamma(\gamma+1)}\|\nu\| t^{\gamma}, \quad t \in I .
\end{aligned}
$$

Since $\|v\| \leq R+\nu(0)$, hence

$\|P \nu(t)-\nu(0)\| \leq L_{1}+\frac{N}{\Gamma(\gamma+1)}(\nu(0)+R) \delta^{\gamma} \leq R$.

Here, $\delta<\left(\left(R-L_{1}\right) \Gamma(\gamma+1) / N(\nu(0)+R)\right)^{1 / \gamma}$.

Case 2: if $P \nu(t)=\psi(t)$ and $-\tau \leq t<0$, then

$|P \nu(t)-\nu(0)|=|\psi(t)-\psi(0)|=|\nu(t)-v(0)| \leq\|\nu(t)-\nu(0)\| \leq R$.

Hence, $P\left(B_{R}\right) \subseteq B_{R}$. The Leray-Schauder fixed point theorem guarantees that operator $P$ has at least one fixed point and so equation (30) has at least one positive solution.

\section{Stability}

In this section, we will discuss local generalized Ulam-Hyers stability and local generalized Ulam-Hyers-Rassias stability for a class of fractional neutral differential equations. For the case $I=[0, T]$ with $f, g: I \times C \longrightarrow \mathbb{R}^{+}$are continuous functions on a closed interval or more generally compact sets, then they are bounded so we can replace the supremum by the maximum. In this case, norm is also called the maximum norm. Let $X=\{x \in C[-\tau, 1]\}$ be a Banach space with $\|x\|=\max _{t \in I}|x(t)|$, where $\tau$ is a nonnegative real number and $z_{t}=z(t+\theta) ;-\tau \leq \theta \leq 0$. We consider the following differential equation:

$$
\begin{cases}{ }^{c} D_{0}^{\gamma}\left[z(t)-g\left(t, z_{t}\right)\right]=z(t) f\left(t, z_{t}\right), & t \in I, \\ z(t)=\psi(t) \geq 0, & t \in[-\tau, 0],\end{cases}
$$

where ${ }^{c} D_{0}^{\gamma}$ is a Caputo derivative with $\gamma \in(0,1)$ and $\psi \in C\left([-\tau, 0], \mathbb{R}^{+}\right)$. We focus on the following inequalities:

$$
\begin{aligned}
& \left\|{ }^{c} D_{0}^{\gamma}\left[u(t)-g\left(t, u_{t}\right)\right]-u(t) f\left(t, u_{t}\right)\right\| \leq \epsilon, \quad t \in I, \\
& \left\|^{c} D_{0}^{\gamma}\left[u(t)-g\left(t, u_{t}\right)\right]-u(t) f\left(t, u_{t}\right)\right\| \leq \psi(t), \quad t \in I .
\end{aligned}
$$

Definition 6 (see [36]). Equation (36) is local generalized Ulam-Hyers stable if there exists a positive real number $c_{1}$ such that, for each positive $\epsilon$ and for every solution $\bar{v} \in C([-\tau, T], \mathbb{R})$ of $(37)$, there exists a solution $\bar{w} \in C([-\tau, T], \mathbb{R}) \quad$ of $\quad(36) \quad$ with $\quad\|\bar{v}(t)-\bar{w}(t)\| \leq$ $c_{1} \epsilon, t \in[-\tau, T]$.

Definition 7 (see [36]). Equation (36) is local generalized Ulam-Hyers-Rassias stable with respect to $\psi$ if there exists $c_{1 \psi}>0$ such that, for every solution $\bar{v} \in C([-\tau, T], \mathbb{R})$ of $(38)$, there exists a solution $\bar{w} \in C([-\tau, T], \mathbb{R})$ of (36) with $\|\bar{v}(t)-\bar{w}(t)\| \leq c_{1 \psi} \psi(t), t \in[-\tau, T]$.

Remark 1. A solution of differential equation is stable (asymptotically stable) if it attracts all other solutions with sufficiently close initial values. On the contrary, in Hyers-Ulam stability, we compare solution of given differential equation with the solution of differential inequality. We say solution of differential equation is stable if it stays close to solution of differential inequality. Hyers-Ulam stability may not imply the asymptotic stability.

Remark 2 (see [37]). A function $\bar{v} \in C([-\tau, T], \mathbb{R})$ is a solution of (37) if and only if there exists $h \in C(I, \mathbb{R})$ such that
(i) $|h(t)| \leq \epsilon, t \in I$
(ii) ${ }^{c} D_{0}^{\gamma}\left[u(t)-g\left(t, u_{t}\right)\right]=u(t) f\left(t, u_{t}\right)+h(t), t \in I$

Similarly, a function $\bar{v} \in C([-\tau, T], \mathbb{R})$ is a solution of (38) if and only if there exists $\widetilde{h} \in C(I, \mathbb{R})$ such that

(i) $|\widetilde{h}(t)| \leq \psi(t), t \in I$

(ii) ${ }^{c} D_{0}^{\gamma}\left[u(t)-g\left(t, u_{t}\right)\right]=u(t) f\left(t, u_{t}\right)+\widetilde{h}(t), t \in I$

Remark 3. Let $0<\gamma<1$ and $\bar{v} \in C([-\tau, T], \mathbb{R})$ be a solution of inequality (37); then, $\bar{v}$ is a solution of the following inequality: 


$$
\left\|\bar{v}(t)-v(0)-g\left(t, \overline{v_{t}}+x_{t}\right)-\frac{1}{\Gamma(\gamma)} \int_{0}^{t}(t-s)^{\gamma-1} \bar{v}(s) f\left(s, \overline{v_{s}}+x_{s}\right) \mathrm{d} s\right\| \leq \frac{t^{\gamma} \epsilon}{\Gamma(\gamma+1)} .
$$

From Remark 2, we have

$$
{ }^{c} D_{0}^{\gamma}\left[u(t)-g\left(t, u_{t}\right)\right]=u(t) f\left(t, u_{t}\right)+h(t), \quad t \in I,
$$

then

$$
\begin{gathered}
u(t)-u(0)+g(0, \psi)-g\left(t, u_{t}\right) \\
=\frac{1}{\Gamma(\gamma)} \int_{0}^{t}(t-s)^{\gamma-1} u(s) f\left(s, u_{s}\right) \mathrm{d} s \\
\quad+\frac{1}{\Gamma(\gamma)} \int_{0}^{t}(t-s)^{\gamma-1} h(s) \mathrm{d} s .
\end{gathered}
$$

Therefore,

$$
\begin{aligned}
u(t)= & u(0)-g(0, \psi)+g\left(t, u_{t}\right) \\
& +\frac{1}{\Gamma(\gamma)} \int_{0}^{t}(t-s)^{\gamma-1} u(s) f\left(s, u_{s}\right) \mathrm{d} s \\
& +\frac{1}{\Gamma(\gamma)} \int_{0}^{t}(t-s)^{\gamma-1} h(s) \mathrm{d} s .
\end{aligned}
$$

By using the same process as in Section 3, i.e., let $x(\cdot):[-\tau, T] \longrightarrow \mathbb{R}^{+}$be the function defined by

$$
x(t)= \begin{cases}\psi(t), & t \in[-\tau, 0], \\ 0, & t \in I .\end{cases}
$$

Therefore, $x_{0}=\psi$ and for every $v \in C\left(I, \mathbb{R}^{+}\right)$with $v(0)=0$. We defined $\bar{v}$ as

$$
\bar{v}(t)= \begin{cases}0, & t \in[-\tau, 0] \\ v(t), & t \in I .\end{cases}
$$

Let $x(\cdot)$ satisfies equation (42). We can decompose $u($.) as $u(t)=\bar{v}(t)+x(t)$, for $0 \leq t \leq T$ so that $u_{t}=\bar{v}_{t}+x_{t}$, for every $0 \leq t \leq T$, where $\bar{v}(t)$ is such that $|\bar{v}(t)|<1, \forall t \in I$ and the function $v(\cdot)$ satisfies

$$
\begin{aligned}
\bar{v}(t)= & v(0)-g(0, \psi)+g\left(t, \overline{v_{t}}+x_{t}\right) \\
& +\frac{1}{\Gamma(\gamma)} \int_{0}^{t}(t-s)^{\gamma-1} \bar{v}(s) f\left(s, \overline{v_{s}}+x_{s}\right) \mathrm{d} s \\
& +\frac{1}{\Gamma(\gamma)} \int_{0}^{t}(t-s)^{\gamma-1} h(s) \mathrm{d} s .
\end{aligned}
$$

By following the conditions in Section 3, we get from (42) that

$$
\begin{aligned}
\bar{v}(t)= & v(0)+g\left(t, \overline{v_{t}}+x_{t}\right) \\
& +\frac{1}{\Gamma(\gamma)} \int_{0}^{t}(t-s)^{\gamma-1} \bar{v}(s) f\left(s, \overline{v_{s}}+x_{s}\right) \mathrm{d} s \\
& +\frac{1}{\Gamma(\gamma)} \int_{0}^{t}(t-s)^{\gamma-1} h(s) \mathrm{d} s .
\end{aligned}
$$

Hence,

$$
\begin{aligned}
\| \bar{v}(t) & -v(0)-g\left(t, \overline{v_{t}}+x_{t}\right) \\
& -\frac{1}{\Gamma(\gamma)} \int_{0}^{t}(t-s)^{\gamma-1} \bar{v}(s) f\left(s, \overline{v_{s}}+x_{s}\right) \mathrm{d} s \| \leq \frac{t^{\gamma} \epsilon}{\Gamma(\gamma+1)} .
\end{aligned}
$$

If $\bar{v} \in C([-\tau, T], \mathbb{R})$ is a solution of inequality (38) then $\bar{v}$ is a solution of the following inequality:

$$
\begin{aligned}
& \| \bar{v}(t)-v(0)-g\left(t, \overline{v_{t}}+x_{t}\right) \\
& -\frac{1}{\Gamma(\gamma)} \int_{0}^{t}(t-s)^{\gamma-1} \bar{v}(s) f\left(s, \overline{v_{s}}+x_{s}\right) \mathrm{d} s \| \\
& \quad \leq \frac{1}{\Gamma(\gamma)} \int_{0}^{t}(t-s)^{\gamma-1} \psi(s) \mathrm{d} s, \\
& \leq \lambda_{\psi} \psi(t), \quad t \in I .
\end{aligned}
$$
as

Before stating stability results, let us take condition (H4)

$(\mathrm{H} 4) c_{2}+\left(T^{\gamma} / \Gamma(\gamma+1)\right)\left(c_{1}+L\right)<1$

Theorem 7. Suppose that (H4) is true and other two conditions

(a) $f, g: I \times C \longrightarrow \mathbb{R}^{+}, h \in C(I, \mathbb{R})$, and $|h(t)| \leq \epsilon, h>0$.

(b) $f$ and $g$ satisfy Lipchitz conditions:

$$
\begin{gathered}
\left\|f\left(t, \overline{v_{t}}+x_{t}\right)-f\left(t, \overline{w_{t}}+x_{t}\right)\right\| \leq c_{1}\left\|\bar{v}_{t}-\bar{w}_{t}\right\|, \\
\left\|g\left(t, \overline{v_{t}}+x_{t}\right)-g\left(t, \overline{w_{t}}+x_{t}\right)\right\|\left\|\leq c_{2}\right\| \bar{v}_{t}-\bar{w}_{t} \|,
\end{gathered}
$$

where $c_{1}$ and $c_{2}$ are nonnegative real numbers holds, then

(i) Equation (36) has a unique solution

(ii) Equation (36) is local generalized Ulam-Hyers stable

Proof. Let $\bar{v} \in C([-\tau, T], \mathbb{R})$ be a unique solution of equation (37). Denote $\bar{w} \in C([-\tau, T], \mathbb{R})$ as the unique solution of the equation

$$
\begin{cases}{ }^{c} D_{0}^{\gamma}\left[z(t)-g\left(t, z_{t}\right)\right]=z(t) f\left(t, z_{t}\right), & t \in I, \\ w(t)=v(t) \geq 0, & t \in[-\tau, 0] .\end{cases}
$$

Then, we have

$$
\begin{aligned}
\bar{w}(t)= & w(0)+g\left(t, \overline{w_{t}}+x_{t}\right) \\
& +\frac{1}{\Gamma(\gamma)} \int_{0}^{t}(t-s)^{\gamma-1} \bar{w}(s) f\left(s, \overline{w_{s}}+x_{s}\right) \mathrm{d} s \\
= & v(0)+g\left(t, \overline{w_{t}}+x_{t}\right) \\
& +\frac{1}{\Gamma(\gamma)} \int_{0}^{t}(t-s)^{\gamma-1} \bar{w}(s) f\left(s, \overline{w_{s}}+x_{s}\right) \mathrm{d} s .
\end{aligned}
$$


We can see that $\|\bar{v}(t)-\bar{w}(t)\|=0$, for $t \in[-\tau, 0]$. For $t \in I$, we obtain

$$
\begin{aligned}
\|\bar{v}(t)-\bar{w}(t)\| & =\left\|\bar{v}(t)-v(0)-g\left(t, \overline{w_{t}}+x_{t}\right)-\frac{1}{\Gamma(\gamma)} \int_{0}^{t}(t-s)^{\gamma-1} \bar{w}(s) f\left(s, \overline{w_{s}}+x_{s}\right) \mathrm{d} s\right\| \\
& \leq \frac{t^{\gamma} \epsilon}{\Gamma(\gamma+1)}+\left\|g\left(t, \overline{v_{t}}+x_{t}\right)-g\left(t, \overline{w_{t}}+x_{t}\right)\right\|+\frac{1}{\Gamma(\gamma)} \int_{0}^{t}(t-s)^{\gamma-1}\left\|\bar{v}(s) f\left(s, \overline{v_{s}}+x_{s}\right)-\bar{w}(s) f\left(s, \overline{w_{s}}+x_{s}\right)\right\| \mathrm{d} s \\
& \leq \frac{t^{\gamma} \epsilon}{\Gamma(\gamma+1)}+c_{2}\left\|\overline{v_{t}}-\overline{w_{t}}\right\|+\frac{c_{1}}{\Gamma(\gamma)} \int_{0}^{t}(t-s)^{\gamma-1}\left\|\bar{v}_{s}-\bar{w}_{s}\right\| \mathrm{d} s+\frac{L}{\Gamma(\gamma)} \int_{0}^{t}(t-s)^{\gamma-1}\left\|\bar{v}_{s}-\bar{w}_{s}\right\| \mathrm{d} s .
\end{aligned}
$$

For $t \in I$, by using

$$
\begin{aligned}
\left\|\bar{v}_{s}-\bar{w}_{s}\right\| & =\max _{0 \leq s \leq t}\left|\bar{v}_{s}-\bar{w}_{s}\right|=\max _{0 \leq s \leq t}|\bar{v}(s+\theta)-\bar{w}(s+\theta)| \\
& =\max _{\theta \leq s+\theta \leq t+\theta}|\bar{v}(s+\theta)-\bar{w}(s+\theta)| \\
& \leq \max _{-\tau \leq s_{1} \leq t}\left|\bar{v}\left(s_{1}\right)-\bar{w}\left(s_{1}\right)\right| \text { where } s+\theta=s_{1}, \quad-\tau \leq \theta<0 \\
& \leq \max _{-\tau \leq s_{1} \leq T}\left|\bar{v}\left(s_{1}\right)-\bar{w}\left(s_{1}\right)\right| .
\end{aligned}
$$

Thus,

$$
\begin{aligned}
\|\bar{v}(t)-\bar{w}(t)\| & \leq \frac{t^{\gamma} \epsilon}{\Gamma(\gamma+1)}+\lambda_{2} \max _{-\tau \leq t_{1} \leq T}\left|\bar{v}\left(t_{1}\right)-\bar{w}\left(t_{1}\right)\right|+\frac{c_{1}}{\Gamma(\gamma)} \int_{0}^{t}(t-s)^{\gamma-1} \\
& \max _{-\tau \leq s_{1} \leq T}\left|\bar{v}\left(s_{1}\right)-\bar{w}\left(s_{1}\right)\right| \mathrm{d} s+\frac{L}{\Gamma(\gamma)} \int_{0}^{t}(t-s)^{\gamma-1} \max _{-\tau \leq s_{1} \leq T}\left|\bar{v}\left(s_{1}\right)-\bar{w}\left(s_{1}\right)\right| \mathrm{d} s \\
& \leq \frac{t^{\gamma} \epsilon}{\Gamma(\gamma+1)}+c_{2}\left\|\bar{v}\left(t_{1}\right)-\bar{w}\left(t_{1}\right)\right\|+\frac{c_{1}+L}{\Gamma(\gamma+1)}\left\|\bar{v}\left(t_{1}\right)-\bar{w}\left(t_{1}\right)\right\| T^{\gamma} \\
& \leq \frac{T^{\gamma} \epsilon}{\Gamma(\gamma+1)}+\left(c_{2}+\frac{T^{\gamma}}{\Gamma(\gamma+1)}\left(c_{1}+L\right)\right)\|\bar{v}(t)-\bar{w}(t)\| .
\end{aligned}
$$

So,

$$
\|\bar{v}(t)-\bar{w}(t)\| \leq c_{1} \epsilon, \text { where } c_{1}=\frac{T^{\gamma}}{\left(1-c_{2}\right) \Gamma(\gamma+1)-\left(c_{1}+L\right) T^{\gamma}} .
$$

Hence, equation (36) is local generalized Ulam-Hyers stable.

Theorem 8. If (H4) is true and

(a) $f, g: I \times C \longrightarrow \mathbb{R}^{+}, \tilde{h} \in C(I, \mathbb{R})$, and $|\widetilde{h}(t)| \leq \psi(t)$, $h>0$.

(b) $f$ and $g$ satisfy Lipchitz conditions:

$$
\begin{aligned}
\left\|f\left(t, \overline{v_{t}}+x_{t}\right)-f\left(t, \overline{w_{t}}+x_{t}\right)\right\| & \leq c_{1}\left\|\bar{v}_{t}-\bar{w}_{t}\right\|, \\
\left\|g\left(t, \overline{v_{t}}+x_{t}\right)-g\left(t, \overline{w_{t}}+x_{t}\right)\right\| & \leq c_{2}\left\|\bar{v}_{t}-\bar{w}_{t}\right\|,
\end{aligned}
$$

where $c_{1}$ and $c_{2}$ are nonnegative real numbers.

(c) The function $\psi \in C\left(I, \mathbb{R}^{+}\right)$is an increasing function and there exists $\lambda_{\psi}>0$ such that

$$
\frac{1}{\Gamma(\gamma)} \int_{0}^{t}(t-s)^{\gamma-1} \psi(s) \mathrm{d} s \leq \lambda_{\psi} \psi(t), \quad t \in I,
$$

holds, then equation (36) is local generalized Ulam-Hyers-Rassias stable with respect to $\psi$. 
Proof. Following the same steps as in Theorem 7, we can find the result, i.e., here we will obtain

$$
\begin{aligned}
\|\bar{v}(t)-\bar{w}(t)\| \leq & \lambda_{\psi} \psi(t)+\lambda_{2} \max _{-\tau \leq t_{1} \leq T}\left|\bar{v}\left(t_{1}\right)-\bar{w}\left(t_{1}\right)\right|+\frac{c_{1}}{\Gamma(\gamma)} \int_{0}^{t}(t-s)^{\gamma-1} \\
& \quad \max _{-\tau \leq s_{1} \leq T}\left|\bar{v}\left(s_{1}\right)-\bar{w}\left(s_{1}\right)\right| \mathrm{d} s+\frac{L}{\Gamma(\gamma)} \int_{0}^{t}(t-s)^{\gamma-1} \max _{-\tau \leq s_{1} \leq T}\left|\bar{v}\left(s_{1}\right)-\bar{w}\left(s_{1}\right)\right| \mathrm{d} s \\
\leq & \lambda_{\psi} \psi(t)+c_{2}\left\|\bar{v}\left(t_{1}\right)-\bar{w}\left(t_{1}\right)\right\|+\frac{c_{1}+L}{\Gamma(\gamma+1)}\left\|\bar{v}\left(t_{1}\right)-\bar{w}\left(t_{1}\right)\right\| T^{\gamma} \\
\leq & \lambda_{\psi} \psi(t)+\left(c_{2}+\frac{T^{\gamma}}{\Gamma(\gamma+1)}\left(c_{1}+L\right)\right)\|\bar{v}(t)-\bar{w}(t)\| .
\end{aligned}
$$

Therefore,

$$
\begin{aligned}
& \|\bar{v}(t)-\bar{w}(t)\| \leq c_{1 \psi} \psi(t) \\
& \text { where } c_{1 \psi}=\frac{\lambda_{\psi} \Gamma(\gamma+1)}{\left(1-c_{2}\right) \Gamma(\gamma+1)-\left(c_{1}+L\right) T \gamma} .
\end{aligned}
$$

Hence, equation (36) is local generalized Ulam-Hyers-Rassias stable.

\section{Example}

In this section, we present an example to explain the applicability of main results.

$$
\begin{aligned}
x(t)-x(t-\tau) & =\frac{1}{\Gamma(1 / 2)} \int_{0}^{t}(t-s)^{(1 / 2)-1} t x(t) \mathrm{d} s-\frac{1}{\Gamma(1 / 2)} \int_{0}^{t}(t-s)^{(1 / 2)-1} x(t-s) \mathrm{d} s \\
x(t) & =x(t-\tau)-\frac{1}{\Gamma(1 / 2)} \int_{0}^{t}(t-s)^{-(1 / 2)} t x(t) \mathrm{d} s-\frac{1}{\Gamma(1 / 2)} \int_{0}^{t}(t-s)^{-(1 / 2)} x(t-s) \mathrm{d} s .
\end{aligned}
$$

Example 1. Consider the problem

$$
\begin{cases}D_{0}^{1 / 2}[x(t)-x(t-\tau)]=t x(t)-x(t-\tau), & t \in[0,1] \\ x(t)=0, & t \in[-\tau, 0] .\end{cases}
$$

One can easily prove that this problem has a unique fixed point and that it has a positive solution, i.e.,

$$
\begin{aligned}
P x(t) & = \begin{cases}x(t-\tau)-\frac{1}{\Gamma(1 / 2)} \int_{0}^{t}(t-s)^{-(1 / 2)} t x(t) \mathrm{d} s-\frac{1}{\Gamma(1 / 2)} \int_{0}^{t}(t-s)^{-(1 / 2)} x(t-s) \mathrm{d} s, & t \in[0,1], \\
0, & t \in[-\tau, 0],\end{cases} \\
\left|P x_{1}(t)-P x_{2}(t)\right| & \leq\left\|x_{1}(t)-x_{2}(t)\right\|+\frac{1}{\Gamma(1 / 2)}\left\|x_{1}(t)-x_{2}(t)\right\| \int_{0}^{t}(t-s)^{-(1 / 2)} \mathrm{d} s+\frac{1}{\Gamma(1 / 2)}\left\|x_{1}(t)-x_{2}(t)\right\| \int_{0}^{t} t(t-s)^{-(1 / 2)} \mathrm{d} s \\
& \leq\left(1+\frac{t^{3 / 2}}{\Gamma(3 / 2)}-\frac{t^{1 / 2}}{\Gamma(1 / 2)}\right)\left\|x_{1}-x_{2}\right\|, \text { with }\left(1+\frac{t^{3 / 2}}{\Gamma(3 / 2)}-\frac{t^{1 / 2}}{\Gamma(1 / 2)}\right) \leq 1, \quad \text { if } t \in[0,1] .
\end{aligned}
$$


For stability, we take (60) as

$$
\begin{cases}D_{0}^{1 / 2}[x(t)-x(t-\tau)]=t x(t)-x(t-\tau), & t \in[0,1], \\ x(t)=z(t), & t \in[-\tau, 0] \\ x(0)=0, & \end{cases}
$$

and the inequalities

$$
\left|D_{0}^{1 / 2}[z(t)-z(t-\tau)]-t z(t)-z(t-\tau)\right| \leq \epsilon_{1}, \quad t \in[0,1],
$$

$\left|D_{0}^{1 / 2}[z(t)-z(t-\tau)]-t z(t)-z(t-\tau)\right| \leq \psi(t), \quad t \in[0,1]$.

To prove that equation (63) is local generalized Ulam-Hyers stable, we take the conditions as in Theorem 7, i.e., a function $z \in C([0,1], \mathbb{R})$ is a solution of inequality (63) if and only if there exists $h \in C([0,1], \mathbb{R})$ such that

$$
\begin{cases}|h(t)| \leq \epsilon_{1}, & t \in[0,1], \\ D_{0}^{1 / 2}[z(t)-z(t-\tau)]=t z(t)-z(t-\tau)+h(t), & t \in[0,1] .\end{cases}
$$

Applying $I_{0^{+}}^{1 / 2}$ on both sides, we obtain

$$
\begin{aligned}
& \| z(t)-z(t-\tau)-\frac{1}{\Gamma(3 / 2)} t^{3 / 2} z(t) \\
& \quad+\frac{1}{\Gamma(1 / 2)} \int_{0}^{t}(t-s)^{-1 / 2} z(t-s) \mathrm{d} s \| \leq \frac{\epsilon}{\Gamma(3 / 2)} .
\end{aligned}
$$

Here, we take unique solution $x(t)$ of equation (63) as

$$
\begin{aligned}
x(t)= & x(t-\tau)+\frac{1}{\Gamma(1 / 2)} \int_{0}^{t}(t-s)^{-1 / 2} t x(t) \mathrm{d} s \\
& -\frac{1}{\Gamma(1 / 2)} \int_{0}^{t}(t-s)^{-1 / 2} x(t-s) \mathrm{d} s, \\
x(t)= & z(t-\tau)+\frac{1}{\Gamma(1 / 2)} \int_{0}^{t}(t-s)^{-1 / 2} t x(t) \mathrm{d} s \\
& -\frac{1}{\Gamma(1 / 2)} \int_{0}^{t}(t-s)^{-1 / 2} x(t-s) \mathrm{d} s .
\end{aligned}
$$

By further simplification, we obtain

$$
\begin{aligned}
\|z(t)-x(t)\| & \leq c_{1} \epsilon ; \\
c_{1} & =\frac{1}{\Gamma(3 / 2)-t^{1 / 2}(t-1)} .
\end{aligned}
$$

Hence, (63) is local generalized Ulam-Hyers stable.

Remark 4. If we replace equation (66) by the inequality,

$$
\begin{cases}|\widetilde{h}(t)| \leq \psi(t), & t \in[0,1], \\ D_{0}^{1 / 2}[z(t)-z(t-\tau)]=t z(t)-z(t-\tau)+\widetilde{h}(t), & t \in[0,1] .\end{cases}
$$

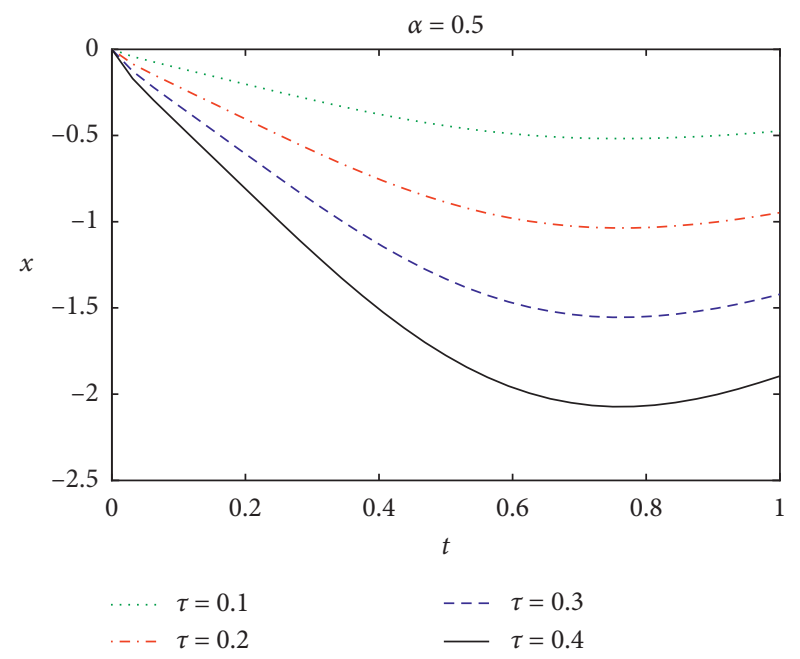

FIgURE 1: Trajectories for different values of $\tau=0.1,0.2,0.3$, and 0.4 with $\alpha=0.5$.

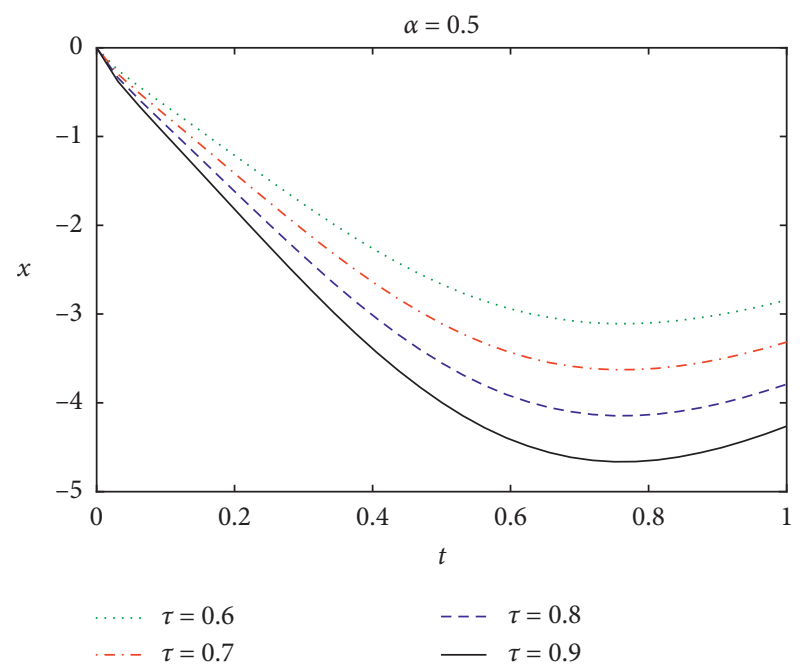

FIgURE 2: Trajectories for different values of $\tau=0.6,0.7,0.8$, and 0.9 with $\alpha=0.5$.

By repeating the same process as in the above example, one can easily verify the main results of Theorem 8 .

\section{Graphical Presentation}

For graphical representation of the solution of the problem given in equation (60), we adopted Adams-Bashforth-Moulton scheme [38] to obtain the numerical solution for this fractional differential equation. To analyze the effect and contribution of time delayed factor, the modified predictor-corrector scheme [39] is incorporated for simulation. To check and demonstrate the stability of the consider model, graphical representation of the solution with different variations of the time delay factor along with other parameters is made. From the numerical results, we could note the UH-stability of the system with varying orders and delays. With the higher orders, the system will achieve the Ulam-Hyers stability more rapidly. It also holds 


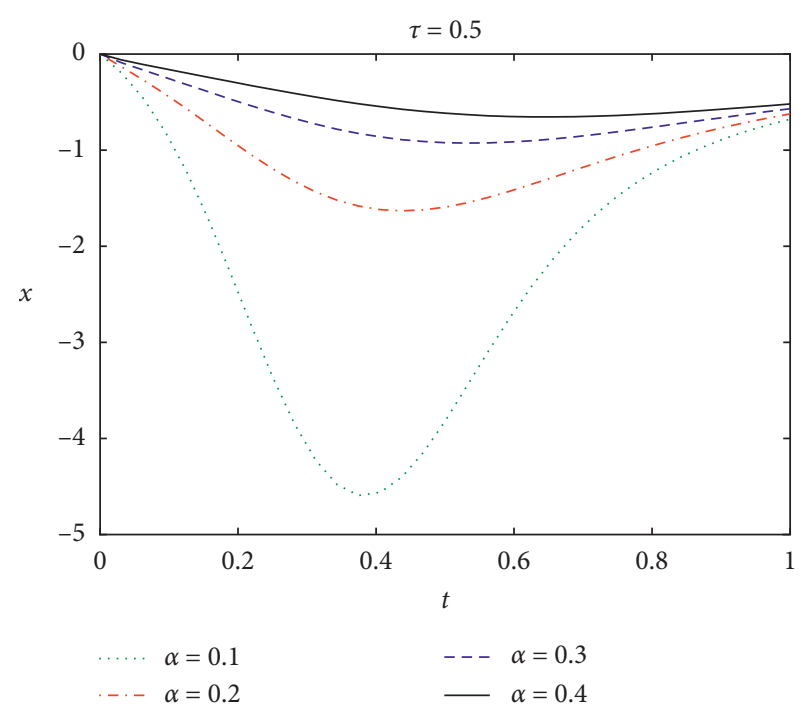

Figure 3: Trajectories for different values of $\alpha=0.1,0.2,0.3$, and 0.4 with time delay $\tau=0.1$.

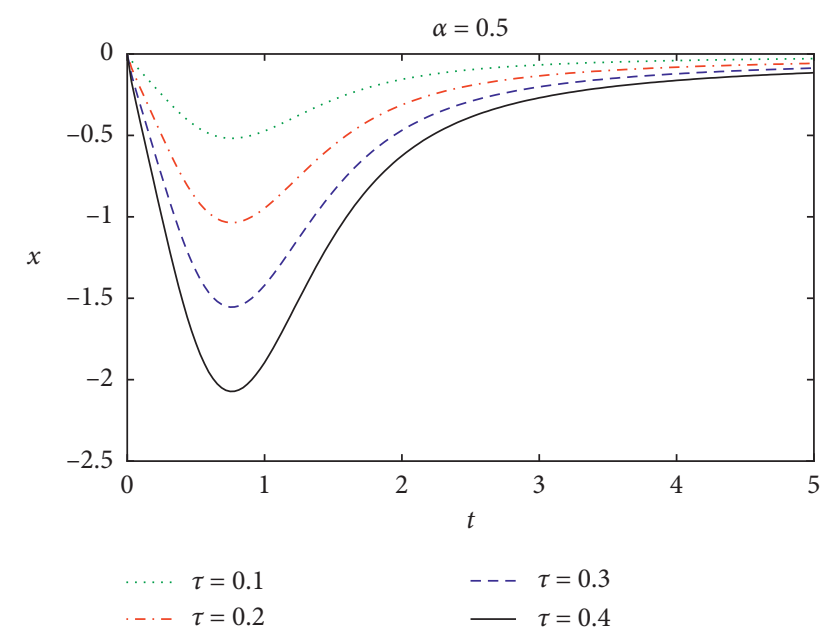

FIGURE 4: Trajectories for different values of $\tau=0.1,0.2,0.3$, and 0.4 with $\alpha=0.5$.

true with increasing delays. One can see the stability of system (60) in Figures (1)-(4) for various values of time delays and fractional derivatives.

\section{Conclusions}

We present some new results about the existence of positive solution for a class of nonlinear fractional differential equations with delay involving Caputo derivative. Leray-Schauder theorem, Arzela-Ascoli theorem, and Banach contraction principle are used for the fixed point property and existence of a solution. We also establish local generalized Ulam-Hyers stability and local generalized Ulam-Hyers-Rassias stability for the same class of nonlinear fractional neutral differential equations. The simulation of an example is also given to show the applicability of our results. The current concepts have significant applications since it means that if we are studying local generalized
Hyers-Ulam-Rassias stable (or Hyers-Ulam stable) system then one does not have to reach the exact solution. We just need to get a function which satisfies a suitable approximation inequality. In other words, local generalized Hyers-Ulam-Rassias stability (or Hyers-Ulam stability) guarantees that there exists a close exact solution. This is altogether useful in many applications where finding the exact solution is quite difficult such as optimization, numerical analysis, biology, and economics. It also helps, if the stochastic effects are small, to use deterministic model to approximate a stochastic one.

\section{Data Availability}

The data used to support the findings of this study are included within the article.

\section{Conflicts of Interest}

The authors declare that they have no conflicts of interest.

\section{Authors' Contributions}

D. Nie, A. U. K. Niazi, and B. Ahmed contributed to and designed the study. A.U.K. Niazi gave the main idea of the manuscript. D. Nie wrote the first draft, and B. Ahmed performed the design of figures. All authors contributed to the manuscript revision, read, and approved the submitted version.

\section{Acknowledgments}

This research is supported by Natural Science Research Project of Anhui Education Department (No. KJ2019A0875); School Level Scientific Research Project of Anhui Xinhua University (No. 2019zr005).

\section{References}

[1] W. G. Glöckle and T. F. Nonnenmacher, "A fractional calculus approach to self-similar protein dynamics," Biophysical Journal, vol. 68, no. 1, pp. 46-53, 1995.

[2] R. Hilfer, Applications of Fractional Calculus in Physics, World Scientific, Singapore, 2000.

[3] A. A. Kilbas, H. M. Srivastava, and J. J. Trujillo, "Theory and applications of fractional differential equations," North-Holland Mathematics Studies, vol. 204, Elsevier, Amsterdam, Netherlands.

[4] X. Li, S. Liu, and W. Jiang, "Positive solutions for boundary value problem of nonlinear fractional functional differential equations," Applied Mathematics and Computation, vol. 217, no. 22, pp. 9278-9285, 2011.

[5] S. Liu, X. Li, X. F. Zhou, and W. Jiang, "Synchronization analysis of singular dynamical networks with unbounded time-delays," Advances in Difference Equations, p. 193, 2015.

[6] J.-B. Liu, J. Zhao, and Z. X. Zhu, "On the number of spanning trees and normalized Laplacian of linear octagonal quadrilateral networks," International Journal of Quantum Chemistry, vol. 119, Article ID 25971, 2019.

[7] J.-B. Liu, J. Zhao, and Z.-Q. Cai, "On the generalized adjacency, Laplacian and signless Laplacian spectra of the 
weighted edge corona networks," Physica A: Statistical Mechanics and its Applications, vol. 540, no. 540, p. 123073, 2020.

[8] J.-B. Liu, J. Zhao, H. He, and Z. Shao, "Valency-based topological descriptors and structural property of the generalized Sierpiński networks," Journal of Statistical Physics, vol. 177, no. 6, pp. 1131-1147, 2019.

[9] J.-B. Liu, Z.-Y. Shi, Y.-H. Pan, J. Cao, M. Abdel-Aty, and U. Al-Juboori, "Computing the Laplacian Spectrum of linear octagonal-quadrilateral networks and its applications," Polycyclic Aromatic Compounds, pp. 1-12, 2020.

[10] K. S. Miller and B. Ross, An Introduction to the Fractional Calculus and Fractional Differential Equations, John Wiley and Sons, Inc., New York, NY, USA, 1993.

[11] I. Podlubny, Fractional Differential Equations, Academic Press, London, UK, 1999.

[12] M. Rehman, R. Khan, and N. Asif, "Three point boundary value problems for nonlinear fractional differential equations," Acta Mathematica Scientia, vol. 31B, no. 4, pp. 13371346, 2011.

[13] A. Babakhani and V. Daftardar-Gejji, "Existence of positive solutions of nonlinear fractional differential equations," Journal of Mathematical Analysis and Applications, vol. 278, no. 2, pp. 434-442, 2003.

[14] V. Daftardar-Gejji, "Positive solutions of a system of nonautonomous fractional differential equations," Journal of Mathematical Analysis and Applications, vol. 302, no. 1, pp. 56-64, 2005.

[15] X. Su, "Positive solutions to singular boundary value problems for fractional functional differential equations with changing sign nonlinearity," Computers \& Mathematics with Applications, vol. 64, no. 10, pp. 3425-3435, 2012.

[16] R. P. Agarwal, Y. Zhou, and Y. He, "Existence of fractional neutral functional differential equations," Computers \& Mathematics with Applications, vol. 59, no. 3, pp. 1095-1100, 2010.

[17] M. Benchohra, J. Henderson, S. K. Ntouyas, and A. Ouahab, "Existence results for fractional order functional differential equations with infinite delay," Journal of Mathematical Analysis and Applications, vol. 338, no. 2, pp. 1340-1350, 2008.

[18] V. Lakshmikantham and A. S. Vatsala, "Basic theory of fractional differential equations," Nonlinear Analysis: Theory, Methods \& Applications, vol. 69, no. 8, pp. 2677-2682, 2008.

[19] V. Lakshmikantham and A. Vatsala, "Theory of fractional differential inequalities and applications," Communications on Pure and Applied Analysis, vol. 11, pp. 395-402, 2007.

[20] V. Lakshmikantham and J. Devi, "Theory of fractional differential equations in Banach space," European Journal of Pure and Applied Mathematics, vol. 1, pp. 38-45, 2008.

[21] V. Lakshmikantham, "Theory of fractional functional differential equations," Nonlinear Analysis: Theory, Methods \& Applications, vol. 69, no. 10, pp. 3337-3343, 2008.

[22] Y. Li and W. Jiang, "Fractional order nonlinear systems with delay in iterative learning control," Applied Mathematics and Computation, vol. 257, pp. 546-552, 2015.

[23] X.-F. Zhou, F. Yang, and W. Jiang, "Analytic study on linear neutral fractional differential equations," Applied Mathematics and Computation, vol. 257, pp. 295-307, 2015.

[24] Y. Li, S. Sun, D. Yang, and Z. Han, "Three-point boundary value problems of fractional functional differential equations with delay," Boundary Value Problems, vol. 2013, no. 1, p. 38, 2013.

[25] Y. Wen, X.-F. Zhou, Z. Zhang, and S. Liu, "Lyapunov method for nonlinear fractional differential systems with delay," Nonlinear Dynamics, vol. 82, no. 1-2, pp. 1015-1025, 2015.
[26] D. Guo and V. Lakshmikantham, Nonlinear Problems in Abstract Cone, Academic Press, Orlando, FL, USA, 1988.

[27] M. Belmekki and K. Mekhalfi, "On fractional differential equations with State-dependent delay via Kuratowski measure of noncompactness," Filomat, vol. 31, no. 2, pp. 451-460, 2017.

[28] K. S. Miller and B. Ross, An Introduction to the Fractional Calculus and Fractional Differential Equations, Wiley, New York, NY, USA, 1993.

[29] S. M. Ulam, A Collection of Mathematical Problems, Interscience Publishers, New York, NY, USA, 1968.

[30] D. H. Hyers, "On the stability of the linear functional equation," Proceedings of the National Academy of Sciences, vol. 27, no. 4, pp. 222-224, 1941.

[31] T. M. Rassias, "On the stability of the linear mapping in Banach spaces," Proceedings of the American Mathematical Society, vol. 72, no. 2, p. 297, 1978.

[32] Y. Zhou, F. Jiao, and J. Li, "Existence and uniqueness for -type fractional neutral differential equations," Nonlinear Analysis: Theory, Methods \& Applications, vol. 71, no. 7-8, pp. 27242733, 2009.

[33] Y. Zhou, F. Jiao, and J. Li, "Existence and uniqueness for fractional neutral differential equations with infinite delay," Nonlinear Analysis: Theory, Methods \& Applications, vol. 71, no. 7-8, pp. 3249-3256, 2009.

[34] J. Wang, M. Feckan, and Y. Zhou, "Ulam's type stability of impulsive ordinary differential equations," Journal of Mathematical Analysis and Applications, vol. 395, no. 1, pp. 258264, 2012.

[35] J. Wang, Y. Zhou, and M. Feckan, "Nonlinear impulsive problems for fractional differential equations and Ulam stability," Computers \& Mathematics with Applications, vol. 64, no. 10, pp. 3389-3405, 2012.

[36] Q. H. Alqifiary and S.-M. Jung, "On the Hyers-Ulam stability of differential equations of Second order," Abstract and Applied Analysis, vol. 2014, pp. 1-8, 2014.

[37] J. Wang, L. Lv, and Y. Zhou, "Ulam stability and data dependence for fractional differential equations with Caputo derivative," Electronic Journal of Qualitative Theory of Differential Equations, vol. 63, no. 63, pp. 1-10, 2011.

[38] K. Diethelm, N. J. Ford, and A. D. Freed, "A predictor-corrector approach for the numerical solution of fractional differential equations," Nonlinear Dynamics, vol. 29, no. 1/4, pp. 3-22, 2002.

[39] S. Bhalekar and V. Daftardar-Gejji, "A predictor-corrector scheme for solving nonlinear delay differential equations of fractional order," Fractional Calculus and Applied Analysis, no. 5, pp. 1-9, 2011. 